\title{
Cross-Sectional Distributions of Platelet-Sized Particles in Blood Flow through Microchannels
}

\author{
Ryota Noso ${ }^{*} \dagger$, Tomoya KIMURA*, Keisuke SAKAMOTO*, \\ Masako SugIHARA-SEKI', and Junji SEKI ${ }^{* *}$ \\ "Department of Pure and Applied Physics, Kansai University, Suita, Osaka 564-8680, JAPAN, \\ ${ }^{* *}$ ORDIST, Kansai University, Suita, Osaka 564-8680, JAPAN \\ (Received : May 5, 2015)
}

\begin{abstract}
In blood flow through microvessels, platelets are known to have enhanced concentrations near the vessel wall, which is the so-called "near-wall excess". In the present study, the spatial distribution of platelet-sized polystyrene particles in red cell suspension flowing through microchannels with a square cross-section of $50 \mu \mathrm{m}$ width was measured by a confocal laser scanning microscope. The measurements were performed at the flow rate of $1.0 \mu \mathrm{L} / \mathrm{min}$ for various particle diameters $(d=1.0-4.8 \mu \mathrm{m})$ and various volume fractions of red cells (hematocrit; Hct $=0-40 \%$ ). In the absence of red cells, the particles were uniformly distributed in the channel cross-section. For all cases studied, their distributions were also uniform at the inlet of the channel. At downstream cross-sections, high concentrations of particles near the channel wall were obtained in the presence of red cells. This tendency was enhanced for higher hematocrits and larger particles. The cross-sectional distribution of the particles revealed enhanced particle probability near the channel corners rather than near the center of the channel faces, suggesting that the particles are focused mainly close to the corners due to the square cross-section of the channel.
\end{abstract}

Key Words: Near-wall excess / Particle distribution / Confocal laser scanning microscope / Red cell suspension

\section{INTRODUCTION}

Platelets act as a player of hemostasis by rapidly accumulating and sticking on the damaged vessel wall. In microcirculation (blood circulation through micrometer-scale blood vessels), platelets are known to have enhanced concentrations near the vessel wall. ${ }^{1,2)}$ This phenomenon is called "near-wall excess" of platelets or "platelet margination". Since this is evidently expedient in platelet functions of hemostasis, there have been intensive studies on the platelet behavior in microvessels, including in vivo and in vitro experiments and numerical model studies. ${ }^{3-8)}$ In general, the Reynolds numbers for the microcirculation are very small.

The spatial distributions of platelet-sized particles in red cell suspension flowing through vessels are affected by several parameters of suspension flow, such as volume concentration of red cells (hematocrit, Hct), red cell deformability, particle size, vessel size, flow rate, and so

\footnotetext{
$\dagger$ Kansai University

Tel: +81 6-6368-1121 ext6509, Fax: +81 6-6368-1121 ext6509,

E-mail:k684372@kansai-u.ac.jp
}

on. In the flow of red cell suspensions, red cells are known to have tendencies to approach the channel centerline due to their high deformability, so that a layer is formed in the vicinity of the vessel wall that is free of red cells. ${ }^{9)}$ When a small amount of platelet-sized particles are mixed in the red cell suspension, the particles are considered to be pushed into the cell free layer near the vessel wall, as a result of the fluid dynamic interaction between red cells and particles. However, this phenomenon of platelet margination has not been fully understood, especially with regard to its dependence on particle size, hematocrit, and red cell deformability.

In the present study, we examined the margination phenomena by in vitro experiments, focusing on the effect of the particle size and the hematocrit. We measured the crosssectional distribution of platelet-sized polystyrene particles in red cell suspensions flowing through microchannels with a square cross-section of $50 \mu \mathrm{m}$ width, utilizing a confocal laser scanning microscope. The particle distributions were compared among different sized particles and among different hematocrits. The developmental change of particle distributions along the flow direction was also discussed. 


\section{MATERIALS AND METHODS}

\subsection{Preparation of Red Blood Cell Suspension}

This study followed the Declaration of Helsinki, and all the subjects provided written informed consent prior to participation. Fresh human blood was sampled from young healthy volunteers. The red cells were washed three times by centrifugation (Model 2410, Kubota, Japan) in phosphatebuffered saline solution (PBS). Red cells were suspended in PBS containing $1 \%$ bovine serum albumin (Wako, Japan) and $5 \%$ dextran (Dextran 60000, Wako, Japan) at desired hematocrits. The resultant hematocrit (Hct) was measured by a microhematocrit technique.

Spherical fluorescent particles with the mean diameter $(d)$ of 1.0, 3.1 and $4.8 \mu \mathrm{m}$ (Thermo Scientific, CA, USA) were added to the red cell suspension at a number concentration of $1.3 \times 10^{4}-6 \times 10^{5} \mathrm{~mm}^{-3}$. The particles are made of polystyrene with density of $1.05 \mathrm{~g} / \mathrm{cm}^{3}$. The excitation and emission maxima of fluorescence are 468 and $508 \mathrm{~nm}$, respectively.

\subsection{Flow System}

Figure 1 shows a schematic of the experimental system used in this study. The flow channel was made of polydimethylsiroxane (PDMS) with rectangular cross-section of $50 \times 50 \mu \mathrm{m}^{2}$ and length of 30 or $50 \mathrm{~mm}$. As shown in Fig. 1(a), the $x$-axis is taken in the flow direction along the centerline of the bottom face of the channel, the $y$-axis is in the horizontal direction, and the $z$-axis is in the vertical direction. The bottom of the channel was sealed with a glass cover slip for observation using an inverted microscope, as shown in Fig. 1(b). Suspension flow was induced by a syringe pump (KDS270, kd Scientific, MA, USA) at a constant flow rate of $1.0 \mu \mathrm{L} / \mathrm{min}$. The mean velocity was $6.7 \mathrm{~mm} / \mathrm{s}$ and the wall shear rate was estimated to be $1070 \mathrm{~s}^{-1}$, which is the same order with the wall shear rate in arterioles of rats. ${ }^{10)}$ The Reynolds number is of the order of 0.1 .

\subsection{Observation and Image Acquisition}

The fluorescent particles flowing through the microchannel were observed with a confocal laser scanning microscope (CLSM) system consisting of an inverted microscope (IX71, Olympus, Japan), a confocal scanning unit (CSU-X1, Yokogawa, Japan), a laser source (Sapphire 488, Coherent, USA) and a high-speed video camera (SV-200i, Photron, Japan) as shown in Fig. 1(b). The confocal unit along with the video camera enabled high-speed image acquisition as high as 1000 frames per second (FPS). By using CLSM, the flow field can be optically sectioned in the direction of optical axis (z-direction in Fig. 1(a)) into thin slices. The images of the flow through the microchannel was recorded at a rate of 500 FPS for 2 - 4 seconds and stored in the fixed disk of PC for later image analysis. Using 40x objective, the recorded image size was $178 \times 178 \mu^{2}$ and the pixel size was $0.348 \times 0.348 \mu \mathrm{m}^{2}$. The observation and recording of the flow was conducted at several distances from the channel inlet $(0 \leq$ $x \leq 30 \mathrm{~mm})$ and at various heights from the bottom $(0<z<$ $25 \mu \mathrm{m})$.

\subsection{Data Analyses: Particle Distributions}

For a micrographic image of suspension flow, the lateral ( $y$ direction) position of each fluorescent particle was measured with use of an image analysis software ImageJ (NIH, USA). We performed this procedure on every 25 frames of all consecutive images recorded at one time (at specified $x$ and $z$ ), and obtained the distribution of the number density of particles along the $y$-axis. This lateral distribution was normalized by the total number of particles recorded at that position, and was represented by a histogram. Its bin width was set to be 2 or $4 \mu \mathrm{m}$. The histogram was symmetrized with regard to the central position $(y=0)$ considering spatial symmetry. When the probability density function (p.d.f.) over the channel crosssection (at specified $x$ ) was concerned, the data of all heights (a)

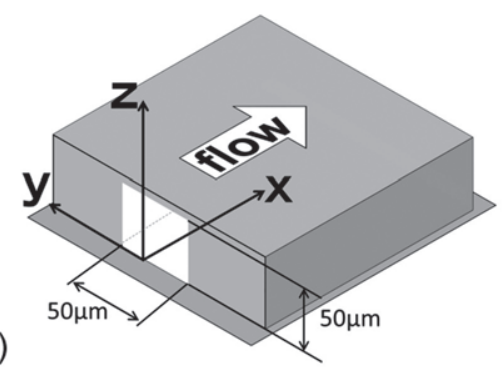

(b)

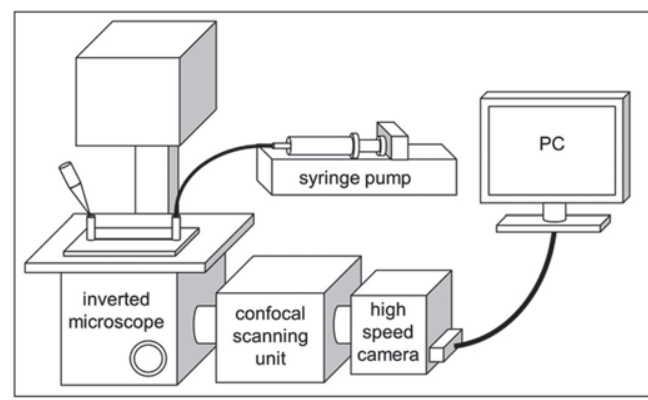

Fig. 1. Schematic of experimental system. (a) Microchannel of $50 \times 50 \mu \mathrm{m}$ cross-section. The $x, y$ and $z$ axes are taken along the flow direction, channel width and channel height, respectively, with the origin at the bottom center of the inlet cross-section. (b) Experimental setup for observation of the flow of red cell suspension with a small amount of platelet-sized fluorescent spherical particles. 
were collected in the $z$-direction and the histogram was further normalized by the total number of particles in the crosssection.

\section{RESULTS AND DISCUSSION}

\subsection{Evolution of Particle Distribution from the Inlet}

As a representative example of the evolution of the particle distribution along the flow direction, Fig. 2 shows the lateral $(y$-) distributions of fluorescent particles at four different locations at (a) $x=0$, (b) 5, (c) 20 and (d) $30 \mathrm{~mm}$. The hematocrit was $20 \%$, the particle diameter was $3.1 \mu \mathrm{m}$, and the height from the bottom face was $z=4 \mu \mathrm{m}$. As seen in Fig. 2(a), the distribution appeared nearly uniform at the channel inlet. Figures 2(b)-(d) show the appearance of the near-wall excess at downstream positions. At $x=5 \mathrm{~mm}$ (Fig. 2(b)), small excess of probability was observed near the channel wall $(y= \pm 25 \mu \mathrm{m})$, and the near-wall excess was enhanced and more prominent at $x=20 \mathrm{~mm}$ (Fig. 2(c)). The probability of particles near the channel wall at $x=$ $30 \mathrm{~mm}$ (Fig. 2(d)) was similar to that at $x=20 \mathrm{~mm}$. Thus, it is suggested that the particle distribution develops with increasing distance from the inlet until it reaches a mature state, which could be realized at a position of $x<20 \mathrm{~mm}$ in this flow condition.

\subsection{Change of Particle Distribution with Height from the Bottom Face}

The variation of the particle distributions with the height from the bottom face is shown in Fig. 3, where the lateral distributions at (a) $z=4$, (b) 12 and (c) $24 \mu \mathrm{m}$ are plotted for Hct $=20 \%, d=3.1 \mu \mathrm{m}$, and $x=20 \mathrm{~mm}$. Figure 3 demonstrates that the probability of particles is large near the channel wall at $z=4$ and $12 \mu \mathrm{m}$, whereas it is close to uniform at $z=24$ $\mu \mathrm{m}$. It is suggested that the near-wall excess is significant near the bottom of the channel cross-section but it is weak near the mid-plane. This is shown more clearly in Fig. 7.

\subsection{Change of Particle Distribution with Hematocrit}

The dependence of the particle distribution on the red cell concentration is shown in Fig. 4, where the lateral distributions are plotted at (a) Hct $=0$, (b) 10, (c) 20, and (d) $40 \%$, for $d=$
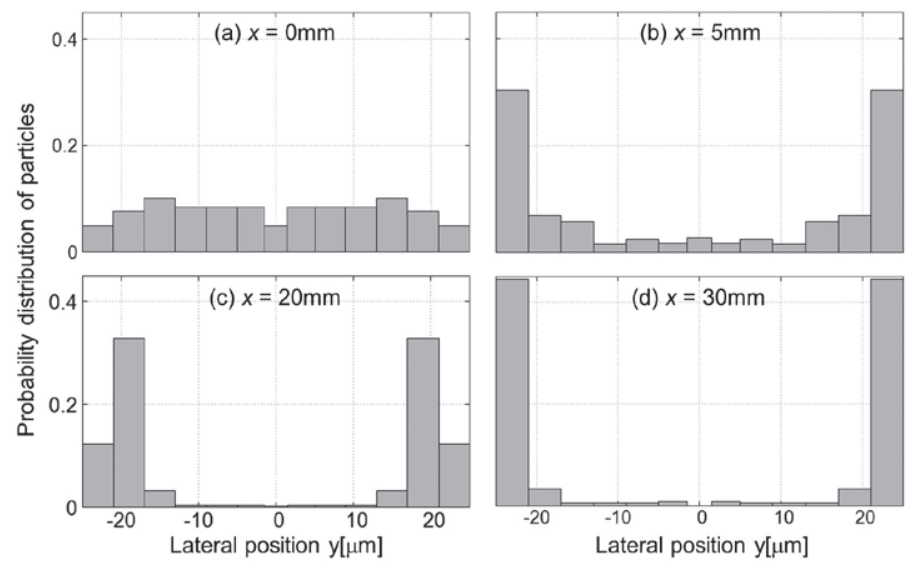

Fig. 2. Variation of lateral distributions of particles with distance from the channel inlet, $x$, from 0 to $30 \mathrm{~mm}$, at $z=4 \mu \mathrm{m}$ for $d=3.1 \mu \mathrm{m}$ and $\mathrm{Hct}=20 \%$.
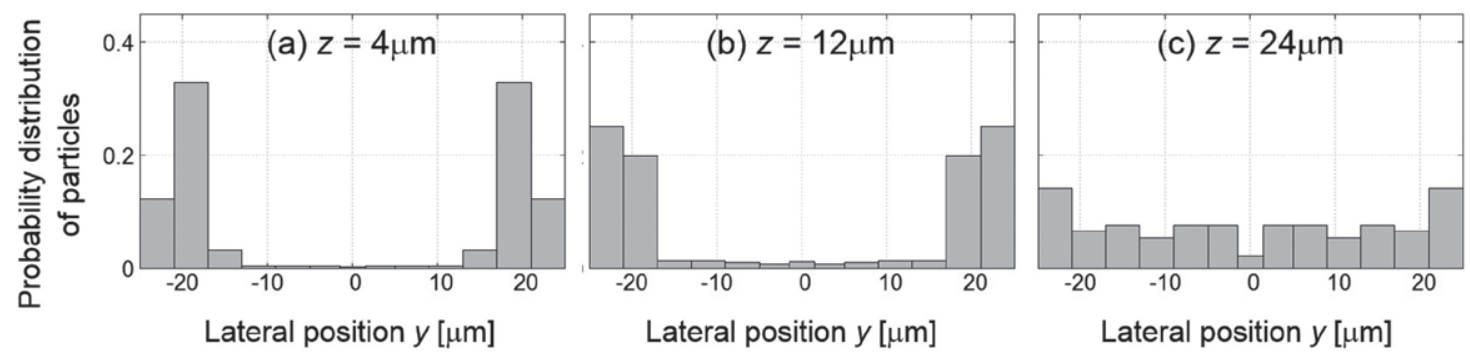

Fig. 3. Variation of particle distribution with height from the bottom face, $z$, from 0 to $24 \mu \mathrm{m}$, at $x=20 \mathrm{~mm}$ for $d=3.1 \mu \mathrm{m}$ and $\mathrm{Hct}=20 \%$. 
$3.1 \mu \mathrm{m}, x=20 \mathrm{~mm}$, and $z=4 \mu \mathrm{m}$. As shown in Fig. 4(a), the particle distribution was almost uniform in the absence of red cells $(\mathrm{Hct}=0 \%)$. This is consistent with the property of low Reynolds number flows that lateral forces do not act on a rigid spherical particle immersed in channel flows of a uniform cross-section. ${ }^{11)}$ In the presence of red cells, on the other hand, the distributions shown in Figs 4(b)-(d) exhibited clearly the near-wall excess. Thus, it can be concluded that the near-wall excess occurs due to the interaction of the particles with red cells. The effect of the hematocrit on the near-wall excess is examined in more detail in section 3.5.

\subsection{Change of Particle Distribution with Particle Diameter}

The lateral distributions of particles with three different diameters $(d=1.0,3.1$ and $4.8 \mu \mathrm{m})$ are compared in Fig. 5, for Hct $=20 \%, x=20 \mathrm{~mm}$, and $z=4 \mu \mathrm{m}$. For $d=1 \mu \mathrm{m}$ (Fig. 5(a)), only slight excess was observed, whereas the near-wall excess was more pronounced for larger particles (Figs 5(b) and (c)).
There are many theoretical model studies concerning particle margination in red cell suspension flow in microchannels. ${ }^{5-8)}$ Among them, 2D- and 3D-model studies ${ }^{8)}$ simulating blood flow in microvessels using dissipative particle dynamics method predicted that the particle margination is more enhanced with larger hematocrit and also with larger particle size, which is coincident with the present results qualitatively.

\subsection{Dependence of Near-Wall Excess on Hematocrit}

In order to express the degree of near-wall excess for each histogram of the particle lateral distribution, we here introduce an index " $R \mathrm{w}$ ", which is defined as the probability of particles residing within the distance of $8 \mu \mathrm{m}$ from the channel wall, which is given by summing the probability of two bins adjacent to the wall in each histogram. The values of $R \mathrm{w}$ range from 0 to 0.5 ; it is 0.16 for the uniform distribution and it is 0.5 when all particles are located within $8 \mu \mathrm{m}$ from the channel wall (maximal near-wall excess).

The variations of $R \mathrm{w}$ with hematocrit are shown in Fig. 6,
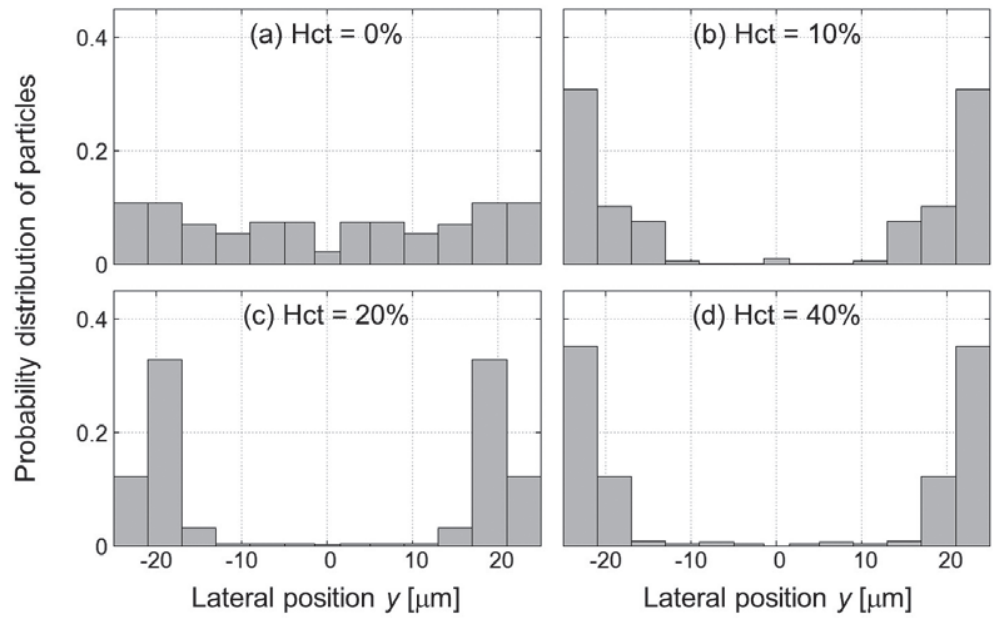

Fig. 4. Variation of particle distribution with hematocrit from 0 to $40 \%$, at $x=20 \mathrm{~mm}$ and $z=4 \mu \mathrm{m}$ for $d=3.1 \mu \mathrm{m}$.
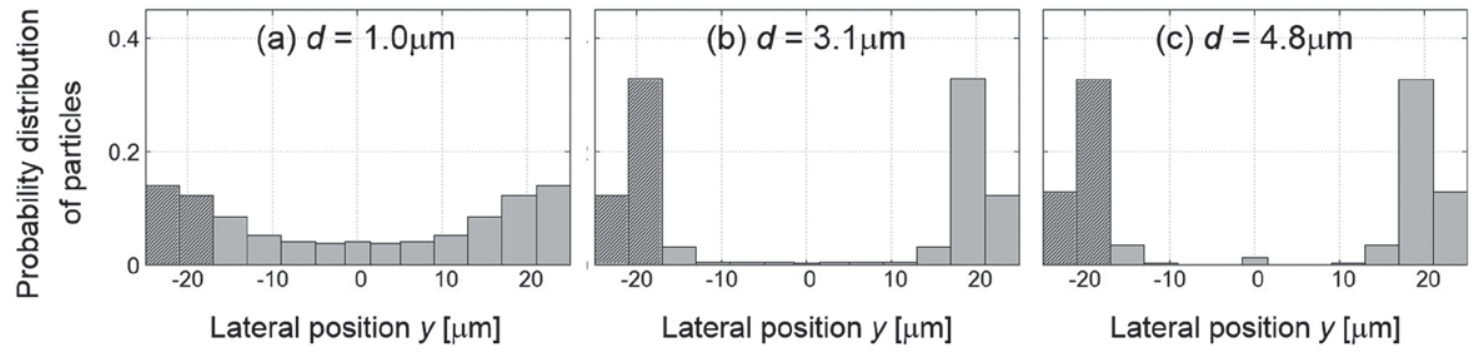

Fig. 5. Variation of particle distribution with particle diameter, $d=1.0,3.1$ and $4.8 \mu \mathrm{m}$, at $x=20 \mathrm{~mm}$ and $z=4 \mu \mathrm{m}$ for Hct $=20 \%$. The summation of the probability in the two hatched bins adjacent to a channel wall represents the index $R \mathrm{w}$. 
for $z=4 \mu \mathrm{m}$ and various particle diameters $(d=1.0,3.1$ and $4.8 \mu \mathrm{m}$ ) at (a) $x=5$ and (b) $20 \mathrm{~mm}$. Both of Figs 6 (a) and (b) show that $R \mathrm{w}$ increases with hematocrit and also with particle diameter. The increasing tendency of $R \mathrm{w}$ with hematocrit is consistent with previous experimental results ${ }^{2)}$ and theoretical prediction. ${ }^{7,8)}$ It is seen from Fig. 6(a) that $R \mathrm{w}$ for $d=3.1$ and $4.8 \mu \mathrm{m}$ is close to the maximum value at Hct $=40 \%$, indicating that these large particles at high Hct attain nearly the maximal near-wall excess state in the distance of as small as $5 \mathrm{~mm}$ from the channel inlet. In contrast, Fig. 6(b) shows that the smallest particles $(d=1.0 \mu \mathrm{m})$ do not reach the maximal near-wall excess state even at $x=20 \mathrm{~mm}$ and Hct $=40 \%$. At an intermediate hematocrit of Hct $=20 \%$, the particles of $d=3.1 \mu \mathrm{m}$ are shown to be nearly in a mature state at $x=20 \mathrm{~mm}$, which is in accord with the results shown in Fig. 2.

\subsection{Cross-Sectional Distribution of Particles}

We constructed the cross-sectional ( $y z-)$ distribution of particles, from the lateral $(y-)$ distributions obtained at every $4 \mu \mathrm{m}$ height from the bottom face $(z=4-24 \mu \mathrm{m})$. Figure 7(a) and (b) show two examples of the cross-sectional distribution for Hct $=20 \%, d=3.1 \mu \mathrm{m}$, at (a) $x=5$ and (b) $20 \mathrm{~mm}$. The upper half of the panels was also drawn for clarity, from symmetry considerations.

Figures 7(a) and (b) demonstrate that the particles are mostly located near the channel corners in the cross-section rather than near the channel wall. The extent of this tendency is larger at the downstream position (Fig. 7(b)) than that at the upstream position (Fig. 7(a)). The feature of particle focusing near the channel corners and their absence near the center of the channel faces results undoubtedly from the geometrical constraint, i.e. square cross-section of the channel. In square
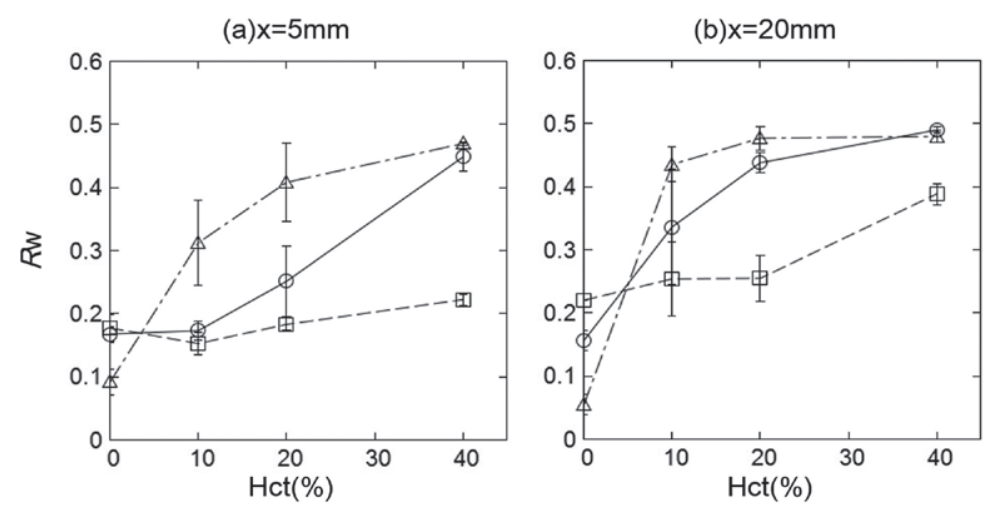

Fig. 6. Degree of near-wall excess, $R \mathrm{w}$ (refer to the definition in the text and Fig. 5), plotted against hematocrit for particle diameters $d=1.0 \mu \mathrm{m}$ (square with broken line), $3.1 \mu \mathrm{m}$ (circle with solid line) and $4.8 \mu \mathrm{m}$ (triangle with dash-dotted line). The distance from the inlet was (a) $x=5 \mathrm{~mm}$ and (b) $20 \mathrm{~mm}$. Marks with error bars represent the mean and the standard deviation.
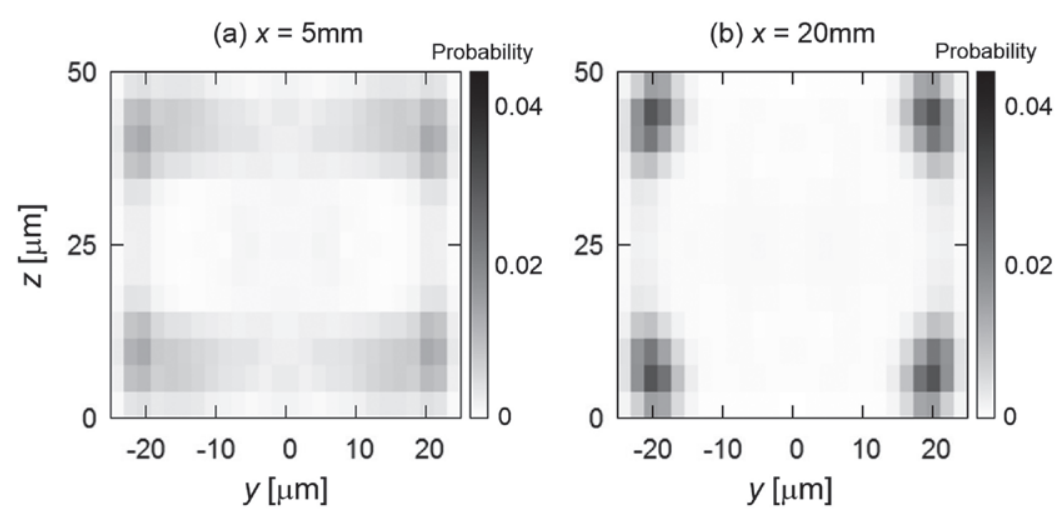

Fig. 7. Cross-sectional probability distributions of particles at (a) $x=5 \mathrm{~mm}$, and (b) $20 \mathrm{~mm}$. The hematocrit was $20 \%$, and the particle diameter was $3.1 \mu \mathrm{m}$. 
channels, red cells may tend to flow in the central region near the channel centerline due to their deformability, which would expel the platelet-sized particles in the outward direction towards the channel wall. Accordingly, the particles may be forced to move into the outermost region of the channel corners. Further studies are required to verify this hypothesis by examining the behaviors of red cells as well.

\section{CONCLUSION}

The spatial distributions of platelet-sized particles were measured in red cell suspension flow through microchannels of square cross-section for various hematocrits and particle diameters. Near-wall excess of the particles was observed for all the cases to a greater or less extent, except for the channel inlet and for red cell free suspension ( $\mathrm{Hct}=0 \%$ ). The degree of near-wall excess was more enhanced with increasing hematocrit as well as increasing particle diameter in the range of $1.0 \leq d \leq 4.8 \mu \mathrm{m}$. The cross-sectional distributions of the particles indicated a particle focusing near four corners of the channel cross-section, which most likely comes from the channel shape.

\section{ACKNOWLEDGEMENTS}

This work was partially supported by JSPS KAKENHI Grant Number 25630057 and ORDIST group grant, Kansai University.

\section{REFERENCES}

1) Tangelder GJ, Teirlinck HC, Slaaf DW, Reneman RS, Amer J Physiol, 248, H318 (1985).

2) Tilles AW, Eckstein EC, Microvasc Res, 33, 211 (1987).

3) Woldhuis B, Tangelder GJ, Slaaf DW, Reneman RS, Amer $J$ Physiol, 262, H1217 (1992)

4) Goldsmith HL, Bell DN, Braovac S, Steinberg A, McIntosh F, Biophys J, 69, 1584 (1995).

5) Crowl LM, Fogelson AL, Int J Numer Method Biomed Eng, 26, 471 (2010)

6) Tokarev AA, Butylin AA, Ermakova EA, Shnol EE, Panasenko GP, Ataullakhanov FI, Biophys J, 101, 1835 (2011).

7) Makino M, Sugihara-Seki M, Biorheology, 50, 149 (2013)

8) Müller K, Fedosov DA, Gompper G, Sci Rep, 4, 4871 (2014).

9) Goldsmith HL, J Gen Physiol, 52, 5 (1968).

10) Seki J, Sasaki Y, Oyama T, Yamamoto J, Biorheology, 33, 463 (1996).

11) Bretherton FP, J Fluid Mech, 24, 284 (1962). 